\title{
Strength post-spinal cord injury: myometer vs manual muscle test
}

\author{
Gerald J Herbison, Zacharia Isaac, Michelle E Cohen and John F Ditunno Jr \\ Department of Rehabilitation Medicine, Jefferson Medical College, Thomas Jefferson University, Philadelphia, \\ Pennsylvania, USA
}

\begin{abstract}
This study was designed to compare changes in strength after spinal cord injury (SCI) with the use of a hand held myometer to the manual muscle test (MMT). Eighty-eight C4-C8 Frankel A-D tetraplegic subjects were tested at various times up to 2 years post-SCI. Elbow flexor strength on successive examinations were grouped according to their early and later MMT scores (3.5 with no change in MMT, 3.5 to 4.0, and 3.5 to 4.5; 4.0 with no change in MMT, 4.0 to 4.5 , and 4.0 to $5.0 ; 4.5$ with no change in MMT, and 4.5 to 5.0). For each group, later myometric measurements (MYO) were expressed as percents of their earlier MYO and were anlayzed using paired Students $t$-tests. Later MYO were 116, 205, 232\% $(P>0.05, P<0.002$, $P<0.05)$ of their earlier MYO for groups 3.5 with no change in the MMT, 3.5 to 4.0 , and 3.5 to 4.5 respectively. Later MYO were $140,139,191 \%(P<0.05, P<0.02, P<0.0001)$ of their earlier MYO for groups 4.0 with no change in MMT, 4.0 to 4.5 , and 4.0 to 5.0 respectively. Later MYO were 127 and $126 \%(P<0.01, P<0.02)$ of their earlier MYO for groups 4.5 with no change in MMT and 4.5 to 5.0 respectively. In conclusion the hand held myometer detected changes in muscle strength not detected by the MMT.
\end{abstract}

Keywords: myometer; manual muscle test; spinal cord injury

\section{Introduction}

Muscle strength assessment is a part of the physical examination of spinal cord injured subjects. Accurate assessment of muscle strength can aid in diagnosis, in detection of improvement or deterioration of the neurological status, and in planning rehabilitation. ${ }^{1}$ The American Spinal Injury Association (ASIA) recommends assessment of muscle strength with the manual muscle test (MMT). ${ }^{2}$ The MMT is the most widely used clinical method of strength assessment. It grades strength according to the ability of a muscle to act against gravity or against resistance offered by an examiner. ${ }^{3-7}$ In studying spinal cord injured tetraplegic subjects, Schwartz et al demonstrated that while MMT scores leveled off with time post-spinal cord injury, myometric measurements (MYO) rose. ${ }^{8}$ The explanation being that in order to receive a grade of 3.0 by the MMT method, only a small fraction of the motor neurons need to be functional while MMT strength grades above a 3.0 require activation of the majority of the remaining neurons. ${ }^{9}$ Quantifying the spectrum of strength into muscle grades subjectively is a relatively insensitive way to measure changes in strength. Beasley found this to be true when he demonstrated that physical therapists using MMT were unable to identify up to $50 \%$ of loss of knee extensor muscle strength in

Correspondence: Gerald J Herbison subjects with poliomyelitis. ${ }^{10}$ The purpose of the present study was to compare changes in strength with the use of the MMT to changes in strength with the use of a hand held myometer.

\section{Methods}

Subjects eligible for admission to this study were admitted to the Regional Spinal Cord Injury Center of the Delaware Valley (RSCICDV) between 1988 and 1993. To be selected for this study, subjects had to have been admitted within $72 \mathrm{~h}$ of injury, had at least two examinations with a hand held myometer and had a minimum 3.5 MMT score for elbow flexors on at least one side. Subjects with manual muscle test scores of 3.0 or lower were not selected for this study because testing elbow flexors with a hand held myometer is not possible for this range of strength unless the extremity is placed in the gravity eliminated position. To maintain consistency, we did not place the arm in the gravity eliminated position to myometrically test muscles with less than 3.5 muscle grades. Subjects had injuries at C4-C8 neurological levels, with Frankel grades A through D. ${ }^{11}$ The subjects were medically stable with no illness, no extremity fractures, and no motivational problems which might preclude accurate measurements. Eighty-eight subjects satisfied these selection criteria, 78 males and 10 females, between ages 15-68 years with an average age of 34 years. 
Subjects who participated in this project were advised of the nature of this study and gave their consent to be studied.

Trained personnel performed the MMT and MYO on each subject. Manual muscle testing was performed by six attending physicians, and three technicians. Everyone had intensive training in performing the MMT when he/she came on the staff of the RSCICDV and two or three times every year thereafter. The three technicians who performed the myometer testing were trained at the outset of the study and three times a year thereafter. We determined ${ }^{12}$ the interrater reliability for the manual muscle and myometer testing was 0.94 and 0.82 respectively. Data collection times post-SCI were determined to be $72 \mathrm{~h} ; 1,2,3$ weeks; and 1, 2, 3, 6, 12, 18 and 24 months post spinal cord injury. Neurological levels as well as impairments were assessed according to American Spinal Injury Association standards. ${ }^{13}$

The ASIA assigned a neural segment between C5T1 to each of five muscle groups in the upper extremity. ${ }^{13}$ The elbow flexors used in this study corresponded to the C5 neural segment. The scale we used for manual muscle strength testing was a 10 grade scale, modified from Brunnstrom and Dennen, ${ }^{14}$ and graded as follows: $0=$ no muscle contraction; $1.0=$ trace muscle contraction; $1.5=$ muscle can perform partial range of motion with gravity eliminated; $2.0=$ muscle can perform full range of motion with gravity eliminated; $2.5=$ muscle can perform full range of motion with gravity eliminated and provides a slight resistance; $3.0=$ muscle can perform full range of motion against gravity; $3.5=$ muscle can perform full range of motion and provides a slight resistance; $4.0=$ the muscle provides considerable resistance; $4.5=$ the muscle provides considerable resistance but not quite normal; $5.0=$ normal muscular strength. ${ }^{14}$ We selected the elbow flexors (C5) for strength assessment in this study because of the large numbers of subjects for whom we had data. The elbow flexors of only one arm were tested in each subject.

The myometric measurements (MYO) were taken with a Penny and Giles (Penny and Giles Transducers Ltd. Dorset, England) hand held myometer with a capacity to measure up to $30 \mathrm{~kg}$. This technique, of measuring strength, was described by Scott, Goddard and Dubowitz. ${ }^{5}$ We have elected to use their designation of strength, as measured in $\mathrm{kg}$, rather than the Systeme International (SI) which defines force in terms of newtons. One newton $(\mathrm{N})$ is the force to impart acceleration of $1 \mathrm{~m}$ per second squared to a mass of $1 \mathrm{~kg}$. Since elbow flexors were tested isometrically, acceleration was zero. For this reason we have reported strength, measured with the myometer, in $\mathrm{kg}$ as reported by Scott and associates. ${ }^{15}$ The strength of either the right or left elbow flexors were measured with this instrument in each subject. Each subject was asked to perform three trials of one to two second maximal contractions with the humerus positioned alongside the body and the elbow flexed 90 degrees. The maximum of the three trials was recorded for the statistical analyses. The myometer was placed on the volar surface of the wrist at the level of the radial styloid and perpendicular to the supinated forearm throughout the test. ${ }^{8}$ Throughout the paper MYO will be used to identify both the singular (myometric measurement) and the plural (myometric measurements).

Manual muscle and myometric testing of strength were not assessed at the same post-SCI time on every subject. Because of this discrepancy, only test times when both MYO and MMT were determined on the same day were included in the analyses. For example, in some subjects 1 week MMT and MYO were determined. Thereafter, the 2 week MMT was performed, but not the MYO. At a subsequent test time, both MMT and MYO were performed and were used in the data analysis. Occasionally, months lapsed before both the MMT and MYO measurements were performed at the same time. Since the study was aimed at comparing myometric with MMT changes in strength, the varying time intervals did not impact on achieving the purpose of the study. The later MYO and MMT were identified as MYO2 and MMT2 respectively, and earlier MYO and MMT were identified as MYO1 and MMT1. Increases in strength were calculated in terms of $\mathrm{MYO} 2$ as percentages of MYO1. To be included as valid data, manual muscle test and myometric values could not fluctuate erratically. They had to either remain constant over two testing intervals or show a steady progression. For example, if a subject had the following successive MMT grades: 3.5, 4.5, 3.5, 4.0, 4.0 , the 4.5 grade was considered an outlier and the

Table 1 Groups

\begin{tabular}{|c|c|c|}
\hline Earlier MMT: 3.5 & Earlier $M M T: 4$ & Earlier $M M T: 4.5$ \\
\hline $\begin{array}{l}\text { A Later MMT } 3.5 \\
\text { ie 3.5-No change }\end{array}$ & $\begin{array}{l}\text { D Later MMT } 4.0 \\
\text { ie } 4-N o \text { change }\end{array}$ & $\begin{array}{l}\text { G Later MMT } 4.5 \\
\text { ie } 4.5-\text { No change }\end{array}$ \\
\hline $\begin{array}{l}\text { B Later MMT } 4.0 \\
\text { ie } 3.5 \text { to } 4.0\end{array}$ & $\begin{array}{l}\text { E Later MMT } 4.5 \\
\text { ie } 4.0 \text { to } 4.5\end{array}$ & $\begin{array}{l}\mathrm{H} \text { Later MMT } 5 \\
\text { ie } 4.5 \text { to } 5\end{array}$ \\
\hline $\begin{array}{l}\text { C Later MMT } 4.5 \\
\text { ie } 3.5 \text { to } 4.5\end{array}$ & $\begin{array}{l}\mathrm{F} \text { Later MMT } 5 \\
\text { ie } 4.0 \text { to } 5\end{array}$ & \\
\hline
\end{tabular}

The MMT graded strength improvements over time are categorized into groups A-H, by their earlier evaluated MMT grade. Each group is further divided into groups according to later MMT grades 
data was assessed as if the two 3.5 scores were successively examined. The MYO and MMT for the 4.5 MMT examination were not included in the analysis.

Myometric compared with MMT changes in strength were analyzed statistically. Successive measurements of strength were grouped as listed in Table 1. Because one or more of these intervals could occur during a subject's 2 year testing period, each subject may have been used in more than one of the groups. For each interval, the later MYO measurement (MYO2) was divided by the earlier (MYO1). The result was multiplied by 100 to obtain a value which expressed the later strength of contraction as a percent of the earlier examination. For example, a subject who had a MMT1 grade of 4.0 and remained a four on MMT2, and had a MYO1 of $3.9 \mathrm{~kg}$ and a MYO2 of $6.0 \mathrm{~kg}$ the second MYO was $154 \%$ of the early examination (or approximately 1.5 times greater than the early examination). When data were exposed as percent change, the geometric mean was the most appropriate measure of central tendency. In order to calculate the geometric mean and coefficients of variation, percent changes were converted to logarithms. The Student $t$-test was employed to compare the second with the first myometric measured value. The geometric means and coefficients of variation of the percent changes were reported.

The mean and coefficient of variation of change in MYO2 as a percent of MYO1, for initial MMT grades of 3.5, 4.0 and 4.5, were calculated and identified in Figures 1 to 3 respectively. For each subject a MYO was used only once for a given initial MMT grade. Only the earlier test values were used in the calculation

\section{\% OF EARLY EXAMINATION}

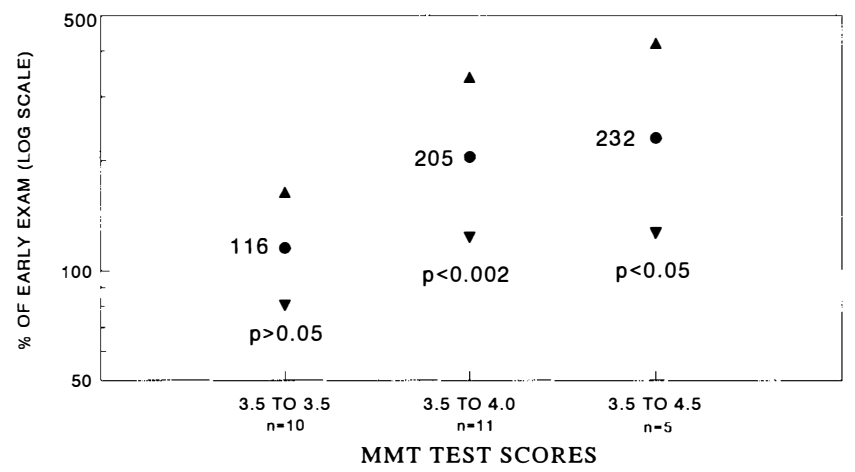

Figure 1 Early MMT1 equal to 3.5. The mean of MYO2 measurements expressed as a percentage of MYO1 measurements are given for intervals where the early MMT examination was 3.5. These percentages, represented on a logarithmic y-axis, are: $116 \%, 205 \%$ and $232 \%$ for the 3.5 No Change, 3.5 to 4.0 , and 3.5 to 4.5 groups respectively. Plus/minus 1 coefficient of variation, represented on the logarithmic $y$-axis, are shown as arrows. The $P$-values indicate significance levels for MYO2 as percents of MYO1 of mean and standard deviation of MYO for the 3.5, 4.0 and 4.5 grade elbow flexor muscles to obtain the results identified in Figure 4.

\section{\% OF EARLY EXAMINATION}

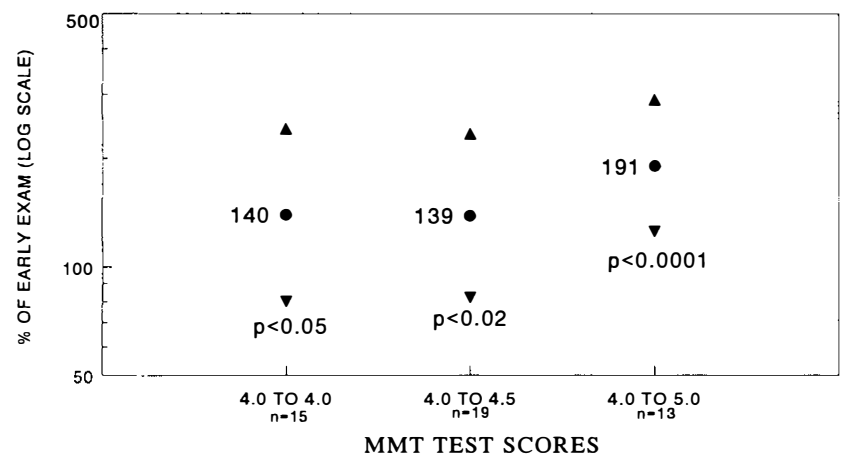

Figure 2 Early MMT1 equal to 4.0. The mean of MYO2 measurements expressed as a percentage of MYO1 measurements are shown for groups with early MMT grades of 4.0. These percentages represented on a logarithmic y-axis are $140 \%, 139 \%$, and $191 \%$ for the $4.0-$ No Change, 4.0 to 4.5 , and 4.0 to 5.0 groups respectively. Plus/minus one coefficient of variation, represented on the logarithmic y-axis, are shown as arrows. The $P$-values indicate significance levels for MYO2 as percents of MYO1

\section{$\%$ OF EARLY EXAMINATION}

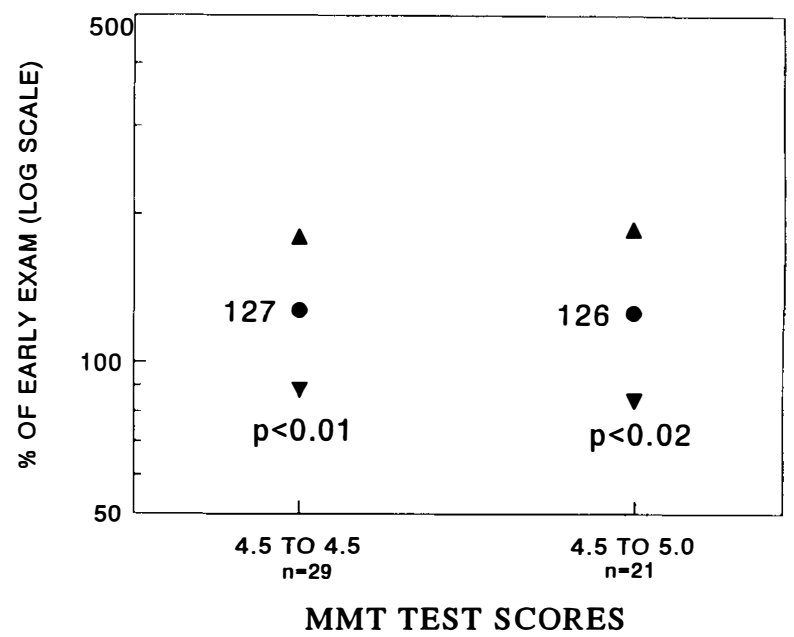

Figure 3 Early MMT1 equal to 4.5. The mean of MYO2 measurements expressed as a percentage of MYO1 measurements are shown for groups with early MMT examination scores of 4.5 . These percentages represented on a logarithmic $y$-axis are: $127 \%$ and $126 \%$ for the 4.5 -No Change, and 4.5 to 5.0 groups respectively. Plus/minus one coefficient of variation, represented on the logarithmic y-axis, are shown as arrows. The $P$-values indicate significance levels for $\mathrm{MYO} 2$ as percents of MYO1 


\section{Results}

Geometric means and coefficients of variation of MYO2 as percents of MYO1, and $P$-values for each group are shown in Table 2 and Figures 1 to 3 . Significant increases in muscle strength, as measured in $\mathrm{kg}$ with the myometer, occurred in Groups D \& G (Table 2) where the MMT remained unchanged (Table 2 and Figures 1-3). As an example, the 4.0-No Change Group (D) did not appear to change with the MMT, whereas with myometry, the MYO2 increased to $243 \%$ of MYO1 (ie mean +1 coefficient of variation). Significant increases in myometric measurements were also found for half grade changes in the MMT (Groups B, E and H), as well as full grade changes in the MMT (Groups $\mathrm{C}$ and F). Figure 4 demonstrates the relationship between myometric measurements and their respective MMT half grades. Myometric measurements, and their coefficients of variations, were greater for each half grade difference in the MMT (Figure 4).

\section{Myometry and MMT Grades}

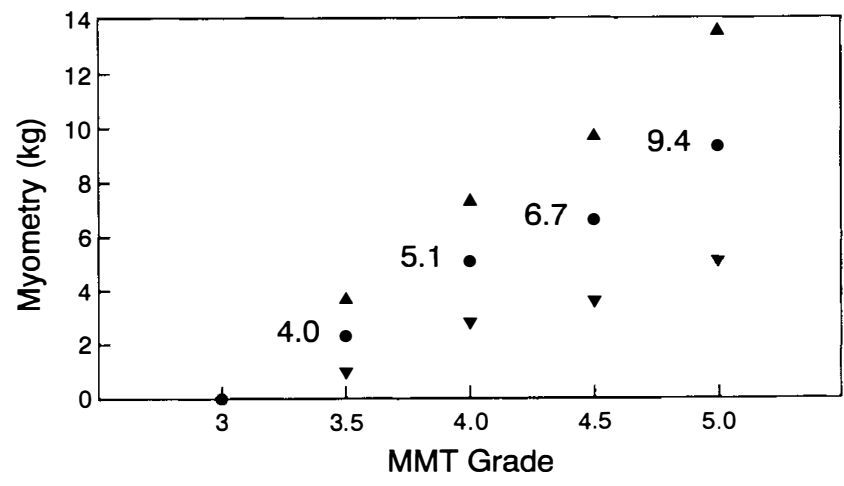

Figure 4 Myometry and MMT grades. MMT score from grades 3.0 to 5.0 are shown with their corresponding mean \pm 1 standard deviation in kilograms $(\mathrm{kg})$. Note that as MMT graded strength increases, so does the mean and standard deviation of myometric measurements

\section{Discussion}

The MMT is recommended for assessing strength by the American Spinal Injury Association ${ }^{13}$ and has been used for clinical research purposes. ${ }^{3-7}$ The results of the present study, however, suggest that changes in strength may be missed when using the MMT. Studies by Waters et al using MMT for strength assessment, suggests that motor strength recovery plateaus 6 months post-SCI. ${ }^{16}$ The findings of the present study demonstrated that changes in strength were detected by a hand held myometer in the absence of changes in the MMT. This suggests that effects of therapeutic interventions, missed by the MMT, might be identified by using a hand held myometer.

Previous studies have demonstrated that a hand held myometer is a useful method of strength assessment. ${ }^{17-20}$ Hand held myometric measurements of strength for various muscle groups have been reported for healthy volunteers ${ }^{21}$ and children with muscular dystrophy. ${ }^{15}$ The values for elbow flexors of healthy male volunteer ${ }^{21}$ were considerably stronger than the mean elbow flexor strength of subjects who had a 5.0 MMT in the present study. This may be because there is a broad range of strength that is considered normal (5.0) by manual muscle testing. In spinal cord injured subjects, strength tested as 5.0 by the MMT may mask a relative decrease in strength compared to the pre-spinal cord injured status of a person. A subject may be strong enough to convince a tester that he/she had a 5.0 MMT grade strength when in fact strength was less than normal compared to the pre-SCI status of the muscle. This supports the value of using a hand held myometer to follow individuals with inflammatory myopathies and motor neuron diseases as reported in some studies. ${ }^{19,20}$

Expressing MYO2 as a percentage of MYO1 may be more easily conceptualized by considering MYO2 as a doubling or tripling of MYO1. As an example, for the 3.5 MMT to 4.0 MMT (Table 2, Group B) the mean MYO2 was $205 \%$ of MYO1. For Group B, MYO2 was approximately two times or a doubling of MYO1 (Figure 1). Likewise, for 4.0 to 5.0 MMT

Table 2 Percent changes in strength identified by interpreting and corresponding change in MMT

\begin{tabular}{|c|c|c|c|c|c|c|}
\hline Group & $N$ & $M M T$ & $G M \%$ & $\begin{array}{c}\text { Coefficient } \\
(G M-1 C V)\end{array}$ & $\begin{array}{l}f \text { variation } \\
(G M+1 C V)\end{array}$ & P-value \\
\hline A & 10 & 3.5 No change & 116 & $\overline{81}$ & 165 & $>0.05$ \\
\hline B & 11 & 3.5 to 4.0 & 205 & 123 & 342 & $<0.002$ \\
\hline $\mathrm{C}$ & 5 & 3.5 to 4.5 & 232 & 127 & 424 & $<0.05$ \\
\hline $\mathrm{D}$ & 15 & 4.0 No change & 140 & 80 & 243 & $<0.05$ \\
\hline E & 19 & 4.0 to 4.5 & 139 & 82 & 234 & $<0.02$ \\
\hline $\mathrm{F}$ & 13 & 4.0 to 5.0 & 191 & 126 & 292 & $<0.001$ \\
\hline $\mathrm{G}$ & 29 & 4.5 No change & 127 & 89 & 180 & $<0.01$ \\
\hline $\mathrm{H}$ & 21 & 4.5 to 5.0 & 126 & 84 & 187 & $<0.02$ \\
\hline
\end{tabular}

The MMT change column identifies the early (MMT1) and later (MMT2) grade strength. The Mean Geometric percent (GM\%) changes of strength show MY O2 measurements as a percent of MY O1 measurement. The Coefficient of Variation (CV) column indicates the Geometric Mean percent changes of strength minus or plus 1 Coefficient of Variation expressed in percent $(\mathrm{GM}-1 \mathrm{CV} \& \mathrm{GM}+1 \mathrm{CV})$. P-values are indicated for each group. Sample size (N) represents the number of measurements used for groups $\mathrm{A}-\mathrm{H}$ 
Group (Table 2, Group F), MYO2 was 191\% of MYO1 or MYO2 was 1.9 times MYO1. Notice the wide variability for the range of improvement within one coefficient of variation in Figures 1-3. The wide variation for $\mathrm{MYO} 2$ measurements expressed as percents of MYO1 measurements suggest that a broad range of strength can be grouped into one MMT grade (Figure 4). The wide variability in the myometric measurement of strength noted in Figure 4 may have been due to differences in myometric testing between testers, but our high interrater reliability (0.82) argues against this possibility.

Gravity serves as a reference for MMT grades of 0 to 3.0, thus making it possible to differentiate objectively between muscle grades. Van der Ploeg and associates reported that only a very small fraction of a muscle functions to give a MMT grade of up to 3.0. ${ }^{9}$ The majority of neurons activate muscle fibers to attain a muscle grade from 3.5 to 5.0 MMT. ${ }^{9}$ Although this range represents the greatest variability in strength, it is also the MMT range in which even trained personnel may have difficulty identifying changes of strength.

Subjects who had grade 4.0 MMT1 and MMT2 had approximately the same myometric strength as subjects who improved one-half grade by the MMT (Table 2). This suggests that half-grade manual muscle testing, between grades 4.0 and 5.0 was not helpful in detecting changes in strength, and supports the American Spinal Injury Association's recommendation of whole grade muscle grading. ${ }^{13}$ However, half grade muscle testing may have clinical value in characterizing differences in strength between arms. Eliminating half grades may either minimize or exaggerate changes in strength as suggested by Figure 4.

The MYO differed approximately fourfold from a MMT of 3.5 compared to 5.0 (Figure 1). This may be of some concern because the American Spinal Injury
Association (ASIA) depicts innervation of a muscle by two nerve segments, and states that by convention a muscle with a grade of 3.0 'is considered to have intact innervation by the most rostral of the innervation segments'. ${ }^{13}$ This suggests that if muscle strength doubled, then the more caudal of two root segments recovered function. The results identified in Figure 1 reflect a fourfold increase in strength for a MMT grade of 3.5 compared to a MMT grade of 5.0. It is difficult to explain a fourfold increase in strength based on recovery of the more caudal of two root segments, and at the same time consider a muscle with a grade of 3.0 to have 'intact innervation by the most rostral of the innervation segments'. Based on van der Ploeg and associates ${ }^{21}$ suggestion that only a very small fraction of muscle contributes to a grade 3.0 and on the results of the present study (Figure 1), a grade 3.0 muscle probably has minimal partial rather than complete innervation by the rostral of the two nerve segments. If as suggested by Beasley, ${ }^{10}$ a MMT of 5.0 could equal $50 \%$ of the strength of a muscle, then an increase in the MMT from 3.5 to 5.0 could reflect full recovery of the rostral root segment rather than any recovery of function of the caudal of the two root segments supplying a muscle. This has implications in interpreting studies that report improvement in root levels after SCI. In summary, the results of the present study suggest that increases in strength, identified by myometric measurement, were masked by manual muscle testing.

\section{Acknowledgements}

This study was supported in part by awards from the National Institute on Disability and Rehabilitation Research to the Regional Spinal Cord Injury Center of Delaware Valley (\#G008535135) and the National Rehabilitation Research and Training Center in Spinal Cord Injury (\#H133B80017).

\section{References}

1 Aitkens $\mathrm{S}$ et al. Relationship of manual muscle testing to objective strength measurements. Muscle Nerve 1989; 12: 173 177.

2 Medical Research Council. Aids to the investigation of peripheral nerve injuries. War Memorandum No 7, 2nd ed. HMSO, London, 1943

3 Marino M, Nicholas JA, Gleim GW. The efficacy of manual assessment of muscle strength using a new device. Am J Sports Med 1982; 10: $360-364$.

4 Mendell JR, Florence J. Manual muscle testing. Muscle Nerve 1990; 13: S16-S20.

5 Brooke $\mathrm{MH}$ et al. Clinical trial in Duchenne dystrophy: the design of the protocol. Muscle Nerve 1981; 4: 186-197.

6 Daniels L, Worthingham C. Muscle Testing Techniques of Manual Examination. WB Saunders, Philadelphia, 1972.

7 Kendall HO, Kendall FP, Wadsworth GE. Muscle, Testing and Function. 2nd ed. Williams \& Wilkins, Baltimore, 1971.

8 Schwartz S, Cohen ME, Herbison GJ, Shah A. Relationship between two measures of upper extremity strength: manual muscle test compared to hand held myometry. Arch Phys Med Rehabil 1992; 73: $1063-1065$.

9 van der Ploeg RJO, Oosterhuis HJGH, Reuvekamp J. Measuring muscle strength. J Neurol 1984; 231: $200-203$.

10 Beasley WC. Quantitative muscle testing: principles and applications to research and clinical services. Arch Phys Med Rehabil 1961; 42: 398 - 425.

11 Frankel HL, Hancock DO, Hystop G. The value of postural reduction in the initial management of closed injuries to the spine with paraplegia and tetraplegia. Paraplegia 1969; 7: 179-192.

12 Cohen ME, Sheehan TP, Herbison GJ. Content validity and reliability of the International Standard for Neurological Classification of Spinal Cord Injury. Top Spinal Cord Injury Rehabil, in press. 
13 American Spinal Cord Injury Association. Standards for neurological classification of spinal injury patients. Chicago, 1989.

14 Brunnstrom F, Dennen M. Round Table on muscle testing. Annual conference of American Physical Therapy Associations. Federation of Crippled and Disabled, Inc. New York: pp. 1-12, 1931.

15 Scott OM, Goddard C, Dubowitz V. Quantitation of muscle strength in children: A prospective study in Duchenne Muscular Dystrophy. Muscle Nerve 1982; 5: 291-301.

16 Waters RL, Adkins RH, Yakura JS, Sie I. Motor and sensory recovery following complete tetraplegia. Arch Phys Med Rehabil 1993; 74: $242-247$.

17 Marciello $\mathrm{M}$ et al. Wrist strength measured by myometry as an indicator of functional independence. $J$ Neurotrauma 1994; 12: $99-105$.
18 Bohannon RW. Hand held myometry. J Neurol Neurosurg Pyschiatry 1992; 55: 982.

19 Moxley RT 3rd. Evaluation of neuromuscular function in inflammatory myopathy. Rheum Dis Clin North Am 1994; 20: $827-843$.

20 Hesselmans LF et al. Evaluation of electrophysiological and clinical tests in an exploratory trial of Org 2766 in motor neuron disease. Neuromuscul Disorders 1993; 3: 319-325.

21 van der Ploeg RJ, Fidler V, Oosterhuis HJ. Hand held myometry: reference values. J Neurol Neurosurg Psychiatry 1991; 54: $244-$ 247. 